\title{
Spectral element method for stability analysis of milling processes with discontinuous time- periodicity
}

Article in International Journal of Advanced Manufacturing Technology · June 2016

Impact Factor: 1.46 · DOI: 10.1007/s00170-016-9044-z

READS

56

4 authors, including:

David Lehotzky

Budapest University of Technology and Eco...

12 PUBLICATIONS 12 CITATIONS

SEE PROFILE
Tamas Insperger

Budapest University of Technology and Eco...

121 PUBLICATIONS 2,778 CITATIONS

SEE PROFILE 
The final publication is available at Springer via http://dx.doi.org/10.1007/s00170-016-9044-z

\title{
Spectral element method for stability analysis of milling processes with discontinuous time-periodicity
}

\author{
David Lehotzky*a, Tamas Insperger ${ }^{\mathrm{a}}$, Firas Khasawneh ${ }^{\mathrm{b}}$, and Gabor Stepan ${ }^{\mathrm{a}}$ \\ ${ }^{a}$ Department of Applied Mechanics, Budapest University of Technology and Economics, Hungary, \\ 1111, Budapest, Muegyetem rkp. 3. \\ ${ }^{\mathrm{b}}$ Department of Engineering, Science and Mathematics, SUNY Polytechnic Institute, 100 Seymour
} Rd Utica, NY 13502, USA

\begin{abstract}
This paper presents an application of the spectral element method for the stability analysis of regenerative machine tool chatter models in milling operations. An extension of the spectral element method is introduced in order to handle the discontinuities in the cutting force in an efficient way. The efficiency of the method is demonstrated on some well-known machine tool chatter models taken from the literature. Efficiency is characterized by the computational time, the convergence of the stability boundaries and the convergence of critical characteristic multipliers. Results show that, compared to the most widespread methods in machining literature, the spectral element method provides significant improvements in computational time while maintaining high accuracy levels.
\end{abstract}

${ }^{*}$ Corresponding author

E-mail address: lehotzky@mm.bme.hu 


\section{Introduction}

Machine tool chatter is the large amplitude vibration between the tool and the workpiece involving intermittent loss of contact. These large amplitude vibrations are harmful to the machining process since they increase the tool wear, result in a poor surface quality or even damage the tool. The most important cause of machine tool chatter is the so called regenerative effect. In many publications chatter is referred to as regenerative chatter. The main point of the regenerative phenomenon is that the cutting force is determined by both the current and the delayed positions of the tool. Therefore, the equations of motion of the machine-tool-workpiece system are governed by a system of delay-differential equations (DDEs).

In Computer Numerical Control (CNC) systems machining parameters are usually selected rather conservatively in order to avoid chatter and its detrimental effects; consequently, CNC systems often utilize sub-optimal machining parameters which results in decreased productivity. This limitation in existing systems has led to a large body of research on increasing productivity while avoiding chatter vibrations. As a result, chatter prediction, avoidance and control has become an important research field and the last decade has seen a steady increase in the number of the corresponding publications [21]. Many of these publications are concerned with identifying the chatter-free regime in the process parameter space. One tool that has been extensively used to illustrate these regimes is the stability lobe diagram.

Stability lobe diagrams (SLDs) chart the domains of technological parameters where the operation is chatter-free or stable, and they guide the selection of optimal technological parameters in order to maximize productivity. In order to increase the reliability of the SLDs and to account for cutting process uncertainties and parameter shifts, several SLDs may need to be reconstructed at different stages of the cutting process. Further, SLDs need to be recalculated when passive 23, 24, 18, or active 14, 19, chatter control strategies are applied. The latter is necessitated by the repeated tuning of the control parameters which requires fast computation of the SLDs.

Consequently, it is important to seek fast algorithms for calculating SLDs. Some of the existing numerical and semi-analytical methods for the stability analysis of machine operations include the multi-frequency solution 1, 2, semi-discretization 19, 11, full-discretization 15, 6, 22, 16, numerical integration [7, Runge-Kutta methods 20, and numerical simulation 25. In many of these methods the primary concern has been the accuracy and the range of application of the approach.

Motivated by the need for taking the speed of the stability calculations into consideration, this paper presents an efficient application of the spectral element (SE) method to the stability analysis of milling processes. In the engineering literature several papers can be found on the spectral element method 12, 13, 14, 15. However, these papers all dealt with systems where the time-varying coefficients are continuous functions of time. In contrast, milling is a system where the time-periodic parameters have discontinuities caused by the periodic entrance and exit of the cutting teeth into the cut. Due to these discontinuities, a further extension of the SE method is needed in order to apply it efficiently to milling models. The difficulties related to the discontinuities and the proper extension of the method are detailed in this paper.

Since in practice it is desirable to apply the most computationally efficient approach, it is necessary to compare the SE method with other well-known methods from the literature. In order to enable the comparison of computational efficiency, the same examples were chosen from the published studies 10, 5, 6, 22, 16, 7, 8, 20. The computational efficiency of the SE method is investigated and compared based on three criteria: the computational time, the convergence of stability boundaries, and the convergence rate of the largest characteristic multiplier.

The paper is organized as follows. First, the mathematical model of machine tool chatter in milling operations is briefly reviewed. Then the SE method is explained and applied to the 
governing equations. Thereafter, an extension of the SE method is explained. This extension handles the discontinuities in the coefficients thus allowing more accurate calculations. The computational properties (computational time, convergence rate of stability boundaries and convergence rate of the largest characteristic multiplier) of the method are then presented. Finally, the results are compared to those of other published methods in the literature.

\section{$2 \quad$ Milling models}

We analyze mechanical models for machine tool chatter in milling processes. Specifically, we study milling with straight fluted tools and uniformly distributed cutting teeth, and we utilize the circular tooth path model. The stability of the variational system is studied around the stationary motions of single- and two-degree-of-freedom (DoF) models. These models are often applied in the machining literature $\underline{\underline{10}}, \underline{\underline{5}}, \underline{\underline{6}}, \underline{\underline{22}}, 16, \underline{\underline{16}}, \underline{\underline{\underline{8}}}, 20$ and they clearly illustrate the proposed extension of the SE method. In this section, the governing equations are given in detail. Note that the derivation of these equations is not described here but can be found in Chapters 5.2.1 and 5.2.4 of [11]. In both the single and two DoF cases, equations determining the stability of the stationary motion are given in the form

$$
\dot{\mathbf{u}}(t)=\mathbf{A} \mathbf{u}(t)-\mathbf{B}(t) \mathbf{u}(t)+\mathbf{B}(t) \mathbf{u}(t-\tau),
$$

where $\mathbf{u} \in \mathbb{R}^{2 m}$, with $m$ being the DoF of the model, $\mathbf{B}(t)=\mathbf{B}(t+\tau)$ for all $t$ time instances and, in the case of a tool with uniformly distributed cutting teeth, the principal period is the tooth passing period $\tau=60 /(\Omega N)$, and matrix $\mathbf{B}(t)$ can be decomposed as

$$
\mathbf{B}(t)=\sum_{r=1}^{N} \mathbf{B}_{r}(t)
$$

The spindle speed of the tool, measured in $\mathrm{rpm}$, is denoted by $\Omega$ and the number of cutting teeth is given by $N$. For the single and two DoF models, state vector $\mathbf{u}(t)$ and matrices $\mathbf{A}$ and $\mathbf{B}_{r}(t)$ are different, as will be shown in the following two subsections.

\subsection{Single DoF model}

For the single DoF model, the state vector and the system matrices are

$$
\mathbf{u}(t)=\left[\begin{array}{c}
\xi(t) \\
\dot{\xi}(t)
\end{array}\right], \mathbf{A}=\left[\begin{array}{cc}
0 & 1 \\
-\omega_{\mathrm{n}}^{2} & -2 \zeta \omega_{\mathrm{n}}
\end{array}\right], \mathbf{B}_{r}(t)=\frac{w K_{\mathrm{n}}}{m_{\mathrm{t}}}\left[\begin{array}{cc}
0 & 0 \\
H_{r}(t) & 0
\end{array}\right],
$$

where $m_{\mathrm{t}}$ is the modal mass, $\zeta$ is the damping ratio and $\omega_{\mathrm{n}}$ is the natural angular frequency of the system, while $w$ denotes the depth of cut and $K_{\mathrm{n}}$ is the normal cutting force coefficient. State variable $\xi(t)$ is a perturbation around the periodic stationary motion $x_{\mathrm{p}}(t)$ of the tool relative to the workpiece, thus the motion of the tool is described by $x(t)=x_{\mathrm{p}}(t)+\xi(t)$. Here, we assume that the vibrations are parallel to the feed and $x$ gives the position of the tip of the tool. The dimensionless specific cutting force coefficient corresponding to tooth $r$ is

$$
H_{r}(t)=g_{r}(t) \sin \left(\varphi_{r}(t)\right)\left(\kappa \cos \left(\varphi_{r}(t)\right)+\sin \left(\varphi_{r}(t)\right)\right)
$$

which is a periodic function with principal period $\tau$. The cutting force coefficient ratio is $\kappa=K_{\mathrm{t}} / K_{\mathrm{n}}$, where $K_{\mathrm{t}}$ denotes the tangential cutting force coefficient. In (4), the functions

$$
\varphi_{r}(t)=(2 \pi \Omega / 60) t+(r-1) 2 \pi / N, \quad r=1,2, \ldots, N
$$


give the angular displacements of the cutting teeth for the case of uniformly distributed cutting teeth and the window function

$$
g_{r}(t)= \begin{cases}1 & \text { if } \varphi_{\mathrm{ent}} \leq \varphi_{r}(t) \bmod 2 \pi \leq \varphi_{\mathrm{ex}} \\ 0 & \text { otherwise }\end{cases}
$$

determines whether the $r^{\text {th }}$ tool is in or out of the cut, respectively. For up-milling, the tool's angle of entrance is $\varphi_{\mathrm{ent}}=0$ and its angle of exit is $\varphi_{\mathrm{ex}}=\arccos \left(1-2 a_{\mathrm{e}} / D\right)$, where $a_{\mathrm{e}}$ is the radial immersion, $D$ is the diameter of the tool, and their ratio $a_{\mathrm{e}} / D$ is the radial immersion ratio. For down-milling, $\varphi_{\mathrm{ent}}=\arccos \left(2 a_{\mathrm{e}} / D-1\right)$ and $\varphi_{\mathrm{ex}}=\pi$.

\subsection{Two DoF model}

In the case of the two DoF model, the state vector and the system matrices are

$$
\mathbf{u}(t)=\left[\begin{array}{c}
\xi(t) \\
\eta(t) \\
\dot{\xi}(t) \\
\dot{\eta}(t)
\end{array}\right], \mathbf{A}=\left[\begin{array}{cc}
\mathbf{0} & \mathbf{I} \\
-\mathbf{K} & -\mathbf{C}
\end{array}\right], \mathbf{B}_{r}(t)=\frac{w K_{\mathrm{n}}}{m_{\mathrm{t}}}\left[\begin{array}{cc}
\mathbf{0} & \mathbf{0} \\
\mathbf{H}_{r}(t) & \mathbf{0}
\end{array}\right]
$$

where

$$
\mathbf{K}=\omega_{\mathrm{n}}^{2} \mathbf{I}, \quad \mathbf{C}=2 \zeta \omega_{\mathrm{n}} \mathbf{I}, \quad \mathbf{H}_{r}(t)=g_{r}(t) \mathbf{T}_{r}(t) \mathbf{K}_{\mathrm{c}} \mathbf{Q}_{r}^{\mathrm{T}}(t),
$$

with $\mathbf{I}$ being a $2 \times 2$ identity matrix and

$$
\mathbf{T}_{r}(t)=\left[\begin{array}{cc}
\cos \left(\varphi_{r}(t)\right) & \sin \left(\varphi_{r}(t)\right) \\
-\sin \left(\varphi_{r}(t)\right) & \cos \left(\varphi_{r}(t)\right)
\end{array}\right], \mathbf{K}_{\mathrm{c}}=\left[\begin{array}{c}
\kappa \\
1
\end{array}\right], \mathbf{Q}_{r}(t)=\left[\begin{array}{c}
\sin \left(\varphi_{r}(t)\right) \\
\cos \left(\varphi_{r}(t)\right)
\end{array}\right]
$$

The motion of the tool is described by the vector

$$
\left[\begin{array}{l}
x(t) \\
y(t)
\end{array}\right]=\left[\begin{array}{l}
x_{\mathrm{p}}(t) \\
y_{\mathrm{p}}(t)
\end{array}\right]+\left[\begin{array}{l}
\xi(t) \\
\eta(t)
\end{array}\right]
$$

where $\xi(t)$ and $\eta(t)$ define a perturbation around the stationary motion given by $x_{\mathrm{p}}(t)$ and $y_{\mathrm{p}}(t)$. Here $x(t)$ and $y(t)$ describe the tool's motion parallel to and perpendicular to the feed velocity, respectively. It is assumed that the modal parameters are the same in $x$ and $y$ directions.

\section{Application of the spectral element method}

The SE method was published in $\underline{12}$ for autonomous delayed systems with a single point delay. Later, the method was extended for autonomous time-delay systems subject to distributed delays 13 and for systems with multiple point delays and time-periodic coefficients 14 . The latest update of the method was carried out in [15, where both distributed delay and multiple point delays were considered with time-periodic coefficients. However, none of the above papers considered time-periodic coefficients with discontinuities, which is the case for milling models described in the previous section. In this section, the SE method is applied to (1) and it is shown that without the special treatment of jumps in the time-periodic coefficients the SE method is inefficient.

The SE method uses the operator equation form of (1), given by

$$
\mathcal{A} \mathbf{u}^{-\tau, \tau}=\mathbf{0}
$$

where

$$
\mathbf{u}^{-\tau, \tau}=\{\mathbf{u}(t): t \in[-\tau, \tau]\}
$$


is a segment of function $\mathbf{u}(t)$ on the interval $t \in[-\tau, \tau]$ and operator $\mathcal{A}$ is defined by

$$
\mathcal{A} \mathbf{u}^{-\tau, \tau}=\{\dot{\mathbf{u}}(t)-\mathbf{A} \mathbf{u}(t)+\mathbf{B}(t) \mathbf{u}(t)-\mathbf{B}(t) \mathbf{u}(t-\tau): t \in[0, \tau]\} .
$$

Note that operator $\mathcal{A}$ gives a mapping between function segments $\mathbf{u}^{-\tau, \tau}$ and $\{\mathbf{0}: t \in[0, \tau]\}$. If an initial function segment $\mathbf{u}^{-\tau, 0}=\{\mathbf{u}(t): t \in[-\tau, 0]\}$ is provided, then the solution segment $\mathbf{u}^{0, \tau}=\{\mathbf{u}(t): t \in[0, \tau]\}$ for interval $t \in[0, \tau]$ can be determined from (11), since $\mathbf{u}^{-\tau, \tau}$ is composed from $\mathbf{u}^{-\tau, 0}$ and $\mathbf{u}^{0, \tau}$ as

$$
\mathbf{u}^{-\tau, \tau}(t)= \begin{cases}\mathbf{u}^{-\tau, 0}(t) & \text { if } t \in[-\tau, 0] \\ \mathbf{u}^{0, \tau}(t) & \text { if } t \in[0, \tau]\end{cases}
$$

Due to the continuity of $\mathbf{u}^{-\tau, \tau}, \mathbf{u}^{-\tau, 0}(0)=\mathbf{u}^{0, \tau}(0)$ also holds.

The SE method splits the operator equation (11) into a system of operator equations by dividing the function segment $\mathbf{u}^{-\tau, \tau}$ into $2 E$ number of elements as

$$
\mathbf{u}_{k}=\left\{\mathbf{u}^{-\tau, \tau}(t): t \in[(k-1) h, k h]\right\}, \quad k=-E+1,-E+2, \ldots, E ;
$$

where $h=\tau / E$ is the length of elements and $E$ is the number of elements over the principal period $\tau$. Due to the continuity of $\mathbf{u}^{-\tau, \tau}$, conditions

$$
\mathbf{u}_{k}(k h)=\mathbf{u}_{k+1}(k h), \quad k=-E+1,-E+2, \ldots, E-1
$$

hold. After the substitution of elements (15) into (11), one obtains the system of operator equations

$$
\mathcal{S} \mathbf{u}_{k}+\boldsymbol{\mathcal { R }}^{k}\left(\mathbf{u}_{k}-\mathbf{u}_{k-E}\right)=\mathbf{0}, \quad k=1,2, \ldots, E ;
$$

where the operators are defined by

$$
\begin{aligned}
\mathcal{S} \mathbf{u}_{k} & = \begin{cases}\dot{\mathbf{u}}_{k}(t)-\mathbf{A} \mathbf{u}_{k}(t) & \text { if } t \in[(k-1) h, k h], \\
\mathbf{0} & \text { otherwise, }\end{cases} \\
\mathcal{R}^{k} \mathbf{u}_{k} & = \begin{cases}\mathbf{B}(t) \mathbf{u}_{k}(t) & \text { if } t \in[(k-1) h, k h], \\
\mathbf{0} & \text { otherwise, }\end{cases} \\
\mathcal{R}^{k} \mathbf{u}_{k-E} & = \begin{cases}\mathbf{B}(t) \mathbf{u}_{k-E}(t-E h) & \text { if } t \in[(k-1) h, k h], \\
\mathbf{0} & \text { otherwise }\end{cases}
\end{aligned}
$$

Using the element-wise coordinate transformation

$$
\zeta_{k}=\frac{2(t-(k-1) h)}{h}-1
$$

and dropping index $k$ of $\zeta$ immediately, operators (18)-(20) become

$$
\begin{aligned}
& \mathcal{S} \mathbf{u}_{k}= \begin{cases}\frac{2}{h} \mathbf{u}_{k}^{\prime}(\zeta)-\mathbf{A} \mathbf{u}_{k}(\zeta) & \text { if } \zeta \in[-1,1] \\
\mathbf{0} & \text { otherwise }\end{cases} \\
& \boldsymbol{\mathcal { R }}^{k} \mathbf{u}_{k}= \begin{cases}\mathbf{B}\left(\frac{h(\zeta+1)}{2}+(k-1) h\right) \mathbf{u}_{k}(\zeta) & \text { if } \zeta \in[-1,1], \\
\mathbf{0} & \text { otherwise }\end{cases} \\
& \boldsymbol{\mathcal { R }}^{k} \mathbf{u}_{k-E}= \begin{cases}\mathbf{B}\left(\frac{h(\zeta+1)}{2}+(k-1) h\right) \mathbf{u}_{k-E}(\zeta) & \text { if } \zeta \in[-1,1], \\
\mathbf{0} & \text { otherwise }\end{cases}
\end{aligned}
$$


Note that the above formulas transform the original problem (1) into the system of operator equations (17), subject to boundary conditions (16).

As a next step, elements $\mathbf{u}_{k}$ are approximated by $\tilde{\mathbf{u}}_{k}$, using element-wise Lagrange interpolation

$$
\tilde{\mathbf{u}}_{k}(\zeta)=\sum_{j=1}^{n+1} \phi_{j}(\zeta) \tilde{\mathbf{u}}_{k, j}
$$

where $\tilde{\mathbf{u}}_{k, j}=\tilde{\mathbf{u}}_{k}\left(\zeta_{j}\right)$ and $\left\{\zeta_{j}\right\}_{j=1}^{n+1}$ is the Legendre-Gauss-Lobatto (LGL) point set (see Appendix A) and the Lagrange base polynomials are given by the barycentric formula

$$
\phi_{j}(\zeta)=\frac{\frac{\varpi_{j}}{\zeta-\zeta_{j}}}{\sum_{l=1}^{n+1} \frac{\varpi_{l}}{\zeta-\zeta_{l}}}, \quad \varpi_{j}=\frac{1}{\omega^{\prime}\left(\zeta_{j}\right)}, \quad \omega(\zeta)=\prod_{j=1}^{n+1}\left(\zeta-\zeta_{j}\right) .
$$

With the substitution of (25), operator equations (17) are not satisfied. Consequently, residual function segments appear in (17) according to

$$
\mathbf{r}_{k}=\mathcal{S} \tilde{\mathbf{u}}_{k}+\boldsymbol{\mathcal { R }}^{k}\left(\tilde{\mathbf{u}}_{k}-\tilde{\mathbf{u}}_{k-E}\right) \neq \mathbf{0}, \quad k=1,2 \ldots, E .
$$

In order to obtain linearly independent algebraic equations for $\tilde{\mathbf{u}}_{k, j}, k=1, \ldots, E ; j=1, \ldots, n+$ 1 ; the SE method takes the inner product of residual functions $\mathbf{r}_{k}(\zeta)$ with test functions $\psi_{i}(\zeta)$ and sets them to zero which results in

$$
\int_{-1}^{1} \mathbf{r}_{k}(\zeta) \psi_{i}(\zeta) \mathrm{d} \zeta=\mathbf{0}, \quad i=1, \ldots, n ; \quad k=1, \ldots, E .
$$

Here the test functions are chosen to be the Legendre base polynomials (see Appendix B). Equations (28) can be expanded as

$$
\sum_{j=1}^{n+1}\left(\mathbf{S}_{i, j} \tilde{\mathbf{u}}_{k, j}+\mathbf{R}_{i, j}^{k}\left(\tilde{\mathbf{u}}_{k, j}-\tilde{\mathbf{u}}_{k-E, j}\right)\right)=\mathbf{0}, \quad i=1, \ldots, n ; \quad k=1, \ldots, E
$$

where

$$
\begin{aligned}
\mathbf{S}_{i, j} & =\int_{-1}^{1}\left(\frac{2}{h} \mathbf{I} \phi_{j}^{\prime}(\zeta)-\mathbf{A} \phi_{j}(\zeta)\right) \psi_{i}(\zeta) \mathrm{d} \zeta \\
\mathbf{R}_{i, j}^{k} & =\int_{-1}^{1} \mathbf{B}\left(\frac{h(\zeta+1)}{2}+(k-1) h\right) \phi_{j}(\zeta) \psi_{i}(\zeta) \mathrm{d} \zeta
\end{aligned}
$$

These integral terms are evaluated numerically using LGL quadrature. Consequently, the quadrature nodes and interpolation nodes are identical and (30)-(31) are calculated according to

$$
\begin{aligned}
\mathbf{S}_{i, j} & =\frac{2}{h} \mathbf{I} \sum_{l=1}^{n+1} F_{i, l} D_{l, j}-\mathbf{A} F_{i, j}, \\
\mathbf{R}_{i, j}^{k} & =F_{i, j} \mathbf{B}_{j}^{k}
\end{aligned}
$$

where

$$
\begin{aligned}
& F_{i, j}=\psi_{i}\left(\zeta_{j}\right) w_{j}, \\
& D_{q, j}=\phi_{j}^{\prime}\left(\zeta_{q}\right)= \begin{cases}\frac{\varpi_{j} / \varpi_{q}}{\zeta_{q}-\zeta_{j}} & \text { if } j \neq q, \\
-\sum_{l \neq 1}^{n+1} \frac{\varpi_{l} / \varpi_{j}}{\zeta_{j}-\zeta_{l}} & \text { if } j=q,\end{cases} \\
& \mathbf{B}_{j}^{k}=\mathbf{B}\left(\frac{h\left(\zeta_{j}+1\right)}{2}+(k-1) h\right)
\end{aligned}
$$




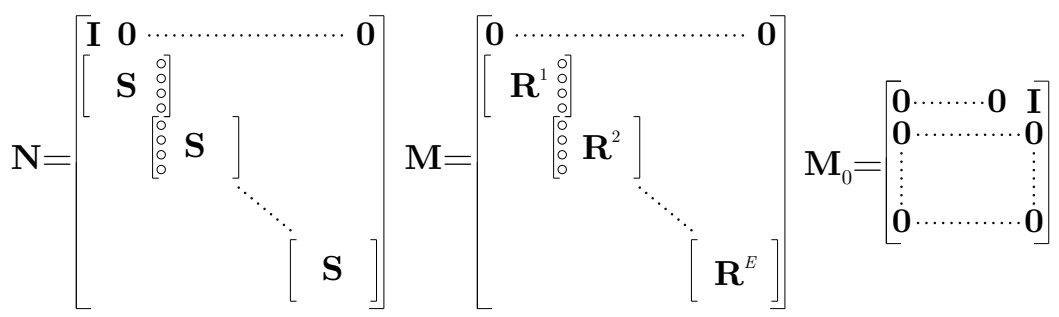

Figure 1: A schematic of the structure of matrices $\mathbf{N}, \mathbf{M}$ and $\mathbf{M}_{0}$ in (37)

and $w_{j}$ is the weight of the LGL quadrature corresponding to $\zeta_{j}$. Note that the LGL quadrature (32) gives exact results for (30); however, (33) is only approximate. Using equations (29) and (16) a mapping can be constructed in the form

$$
(\mathbf{N}+\mathbf{M}) \mathbf{X}_{\tau}=\left(\mathbf{M}+\mathbf{M}_{0}\right) \mathbf{X}_{0}
$$

where

$$
\mathbf{X}_{0}=\left[\begin{array}{l}
\tilde{\mathbf{u}}_{-E+1,1} \\
\tilde{\mathbf{u}}_{-E+1,2} \\
\vdots \\
\tilde{\mathbf{u}}_{-E+1, n+1} \\
\tilde{\mathbf{u}}_{-E+2,2} \\
\vdots \\
\tilde{\mathbf{u}}_{-E+2, n+1} \\
\tilde{\mathbf{u}}_{-E+3,2} \\
\vdots \\
\vdots \\
\tilde{\mathbf{u}}_{0, n} \\
\tilde{\mathbf{u}}_{0, n+1}
\end{array}\right], \quad \mathbf{X}_{\tau}=\left[\begin{array}{l}
\tilde{\mathbf{u}}_{1,1} \\
\tilde{\mathbf{u}}_{1,2} \\
\vdots \\
\tilde{\mathbf{u}}_{1, n+1} \\
\tilde{\mathbf{u}}_{2,2} \\
\vdots \\
\tilde{\mathbf{u}}_{2, n+1} \\
\tilde{\mathbf{u}}_{3,2} \\
\vdots \\
\vdots \\
\tilde{\mathbf{u}}_{E, n} \\
\tilde{\mathbf{u}}_{E, n+1}
\end{array}\right]
$$

and the structure of matrices $\mathbf{N}, \mathbf{M}$ and $\mathbf{M}_{0}$ is shown in Figure 1 . The $2 m n \times 2 m(n+1)$ dimensional submatrices $\mathbf{S}$ and $\mathbf{R}^{k}$ are composed from elements (32)-(33) as

$$
\mathbf{S}=\left[\mathbf{S}_{i, j}\right]_{i, j=1}^{n, n+1}, \quad \mathbf{R}^{k}=\left[\mathbf{R}_{i, j}^{k}\right]_{i, j=1}^{n, n+1}
$$

In Figure 1, the blank locations of the matrices represent entries of zero, and sub-matrices $\mathbf{R}^{k+1}$ are shifted to the right relative to sub-matrices $\mathbf{R}^{k}$ with $2 m n$ columns. The resulting horizontal overlap is indicated by circles in the figure. Figure 1 shows the same structure for sub-matrices $\mathbf{S}$ which constitute the majority of matrix $\mathbf{N}$. Using mapping (37), the approximation of the monodromy operator can be calculated as

$$
\mathbf{U}=(\mathbf{N}+\mathbf{M})^{-1}\left(\mathbf{M}+\mathbf{M}_{0}\right)
$$

The stability of the system is determined by $\mu_{\mathrm{cr}}$ that is the largest-in-modulus eigenvalue of $\mathbf{U}$. If $\left|\mu_{\mathrm{cr}}\right| \leq 1$ then the approximate system (37) is stable, otherwise it is unstable.

Note that during the calculation of the stability maps, integral terms (31) have to be recalculated each time $\Omega$ is updated (which is time-consuming) because they contain $\Omega$ via (5). In order to eliminate the dependence of (31) on $\Omega$, the angle domain with variable $s=2 \pi \Omega t / 60$ is used in the sequel instead of the time domain with variable $t$. This transforms (1) to

$$
\mathbf{u}^{\prime}(s)=\tilde{\mathbf{A}} \mathbf{u}(s)-\tilde{\mathbf{B}}(s) \mathbf{u}(s)+\tilde{\mathbf{B}}(s) \mathbf{u}(t-2 \pi / N),
$$




\begin{tabular}{|c|l|}
\hline$m_{\mathrm{t}}$ & $0.03993 \mathrm{~kg}$ \\
\hline$\omega_{\mathrm{n}}$ & $922 \times 2 \pi \mathrm{rad} / \mathrm{s}$ \\
\hline$\zeta$ & 0.011 \\
\hline$K_{\mathrm{t}}$ & $6 \times 10^{8} \mathrm{~N} / \mathrm{m}^{2}$ \\
\hline$K_{\mathrm{n}}$ & $2 \times 10^{8} \mathrm{~N} / \mathrm{m}^{2}$ \\
\hline$N$ & 2 \\
\hline
\end{tabular}

Table 1: Parameters used throughout the paper, chosen according to $\underline{10}$ where now $\square^{\prime}$ denotes differentiation with respect to $s$ and

$$
\tilde{\mathbf{B}}(s)=\sum_{r=1}^{N} \tilde{\mathbf{B}}_{r}(s)
$$

with matrices

$$
\tilde{\mathbf{A}}=\left[\begin{array}{cc}
0 & 1 \\
-1 / \Omega_{\mathrm{d}}^{2} & -2 \zeta / \Omega_{\mathrm{d}}
\end{array}\right], \tilde{\mathbf{B}}_{r}(s)=\frac{w_{\mathrm{d}}}{\Omega_{\mathrm{d}}^{2}}\left[\begin{array}{cc}
0 & 0 \\
H_{r}(s) & 0
\end{array}\right]
$$

and

$$
\tilde{\mathbf{A}}=\left[\begin{array}{cc}
\mathbf{0} & \mathbf{I} \\
-\frac{1}{\Omega_{\mathrm{d}}^{2}} \mathbf{I} & -\frac{2 \zeta}{\Omega_{\mathrm{d}}} \mathbf{I}
\end{array}\right], \tilde{\mathbf{B}}_{r}(s)=\frac{w_{\mathrm{d}}}{\Omega_{\mathrm{d}}^{2}}\left[\begin{array}{cc}
\mathbf{0} & \mathbf{0} \\
\mathbf{H}_{r}(s) & \mathbf{0}
\end{array}\right]
$$

for the single and two DoF models, respectively. The dimensionless spindle speed is denoted by $\Omega_{\mathrm{d}}=2 \pi \Omega /\left(60 \omega_{\mathrm{n}}\right)$ while the dimensionless depth of cut is $w_{\mathrm{d}}=w /\left(m_{\mathrm{t}} \omega_{\mathrm{n}}^{2}\right)$. After dropping the tildes, the SE method is applied using the formulas above with dimensionless delay $\tau=2 \pi / N$.

Throughout this paper, the results are presented for the parameter set given in Table 1. For down-milling, the results are shown in Figure 2. The left column of the figure shows that the low immersion ratio $\left(a_{\mathrm{e}} / D=0.05\right)$ results in periodic jumps in $H(s)=\sum_{r=1}^{N} H_{r}(s)$ and that the convergence of the stability chart is very slow. Even with polynomial order $n=50$, the stability boundary does not coincide with the exact one. In contrast, the right column of Fig. 2 shows that in the case of full immersion $\left(a_{\mathrm{e}} / D=1\right)$ there is no discontinuity in $H(s)$ and that the convergence of the stability boundary is fast. In this case polynomial order $n=25$ already gives accurate results. It can be thus inferred that stability maps converge slowly when discontinuities are present in $H(s)$ (that is when $\mathbf{B}(s)$ is discontinuous), otherwise they converge fast. Consequently, in order to avoid poor convergence properties it is necessary to further improve the method to handle discontinuities. These improvements are detailed in the next section.

\section{Extension for handling discontinuities}

The inaccuracy in the stability calculation is caused by the inaccuracy of the LGL quadrature for term (31) due to the discontinuity of function $\mathbf{B}(s)$. In order to avoid discontinuity within the integral term (31), the domain of integration is split into sub-domains where the $\mathbf{B}(s)$ function remains smooth. Discontinuities are caused either by the entering or the exiting of the tool. Consequently, in order to locate the discontinuities, it is useful to decompose $\mathbf{B}(s)$ into parts that correspond to different teeth. This leads to the expression

$$
\mathbf{R}_{i, j}^{k}=\sum_{r=1}^{N} \mathbf{R}_{i, j}^{k, r}=\sum_{r=1}^{N} \int_{-1}^{1} \mathbf{B}_{r}\left(\frac{h(\eta+1)}{2}+(k-1) h\right) \phi_{j}(\eta) \psi_{i}(\eta) \mathrm{d} \eta .
$$





Figure 2: Convergence of stability maps for $m=1$ DoF, down-milling without any treatment of discontinuities in $\mathbf{B}(s)$. The radial immersion ratios are $a_{\mathrm{e}} / D=0.05$ and $a_{\mathrm{e}} / D=1$, the element number is $E=1$ and the stability maps are calculated on a $400 \times 400$ grid. Further parameters are taken from Table 1.

The discontinuity points can be located for each $\mathbf{B}_{r}(s)$ function. In this paper, we analyze milling tools with uniformly distributed cutting teeth. Let us denote the angle of rotation corresponding to the entrance and exit of the $r$-th tooth by

$$
s_{1}^{r}=\varphi_{\mathrm{ent}}-(r-1) \frac{2 \pi}{N}, \quad s_{2}^{r}=\varphi_{\mathrm{ex}}-(r-1) \frac{2 \pi}{N},
$$

respectively. The possible cases for the location of the discontinuities relative to the principal period $[0, \tau]$ are the following:

I) $0<s_{1}^{r}<\tau$ and $0<s_{2}^{r}<\tau$

$$
\begin{array}{ll}
\delta_{1}^{r}=s_{1}^{r} \bmod h, & q_{1}^{r}=\operatorname{int}\left(s_{1}^{r} / h\right)+1 \\
\delta_{2}^{r}=s_{2}^{r} \bmod h, & q_{2}^{r}=\operatorname{int}\left(s_{2}^{r} / h\right)+1
\end{array}
$$

II) $s_{1}^{r} \leq 0$ and $0<s_{2}^{r}<\tau$

$$
\begin{aligned}
\delta_{1}^{r}=0, & q_{1}^{r}=1 \\
\delta_{2}^{r}=s_{2}^{r} \bmod h, & q_{2}^{r}=\operatorname{int}\left(s_{2}^{r} / h\right)+1
\end{aligned}
$$

III) $0<s_{1}^{r}<\tau$ and $s_{2}^{r} \geq \tau$

$$
\begin{aligned}
\delta_{1}^{r}=s_{1}^{r} \bmod h, & q_{1}^{r}=\operatorname{int}\left(s_{1}^{r} / h\right)+1 \\
\delta_{2}^{r}=h, & q_{2}^{r}=E
\end{aligned}
$$

IV) $s_{1}^{r} \leq 0$ and $s_{2}^{r} \geq \tau$

$$
\begin{array}{ll}
\delta_{1}^{r}=0, & q_{1}^{r}=1 \\
\delta_{2}^{r}=h, & q_{2}^{r}=E
\end{array}
$$

V) $s_{1}^{r} \leq 0$ and $s_{2}^{r} \leq 0$ (the $r^{\text {th }}$ tooth is not in the cut)

$$
\begin{array}{ll}
\delta_{1}^{r}=0, & q_{1}^{r}=1 \\
\delta_{2}^{r}=0, & q_{2}^{r}=1
\end{array}
$$




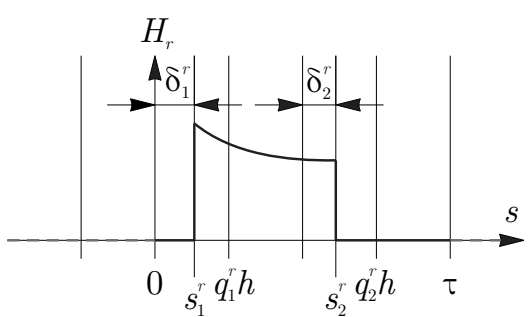

I)

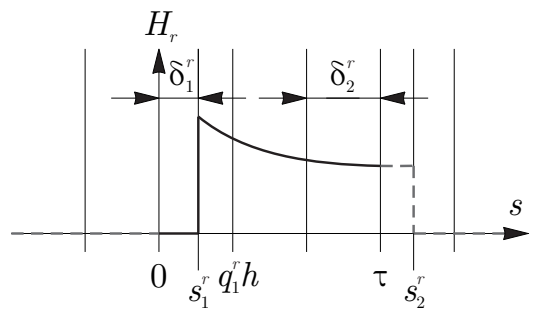

III)

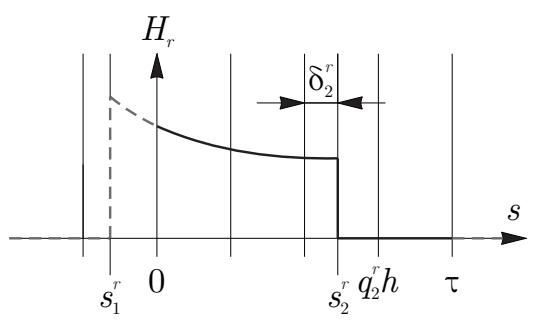

II)

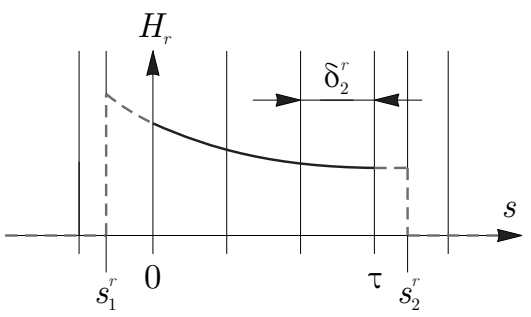

IV)

Figure 3: Different scenarios for the relation between the principal period and the entrance and exit of the $r^{\text {th }}$ tooth, which define the discontinuities in $H_{r}(s)$.

VI) $s_{1}^{r} \geq \tau$ and $s_{2}^{r} \geq \tau$ (the $r^{\text {th }}$ tooth is not in the cut)

$$
\begin{array}{ll}
\delta_{1}^{r}=h, & q_{1}^{r}=E \\
\delta_{2}^{r}=h, & q_{2}^{r}=E
\end{array}
$$

Only the first four cases are associated to actual cutting over the principal period $[0, \tau]$, these cases are illustrated in Figure 3. In the above formulas int $(\cdot)$ denotes the integer part. Using these formulas, the integral terms in (45) can be calculated as

$$
\mathbf{R}_{i, j}^{k, r}= \begin{cases}\int_{-1}^{1} \mathbf{B}_{r}\left(\frac{h(\eta+1)}{2}+(k-1) h\right) \phi_{j}(\eta) \psi_{i}(\eta) \mathrm{d} \eta & \text { if } q_{1}^{r}<k<q_{2}^{r}, \\ \int_{-1+\beta_{1}^{r}}^{1} \mathbf{B}_{r}\left(\frac{h(\eta+1)}{2}+(k-1) h\right) \phi_{j}(\eta) \psi_{i}(\eta) \mathrm{d} \eta & \text { if } k=q_{1}^{r} \neq q_{2}^{r}, \\ \int_{-1}^{-1+\beta_{2}^{r}} \mathbf{B}_{r}\left(\frac{h(\eta+1)}{2}+(k-1) h\right) \phi_{j}(\eta) \psi_{i}(\eta) \mathrm{d} \eta & \text { if } k=q_{2}^{r} \neq q_{1}^{r}, \\ \int_{-1+\beta_{1}^{r}}^{-1+\beta^{r}} \mathbf{B}_{r}\left(\frac{h(\eta+1)}{2}+(k-1) h\right) \phi_{j}(\eta) \psi_{i}(\eta) \mathrm{d} \eta & \text { if } k=q_{1}^{r}=q_{2}^{r}, \\ \mathbf{0} & \text { otherwise, }\end{cases}
$$

where $\beta_{1}^{r}=2 \delta_{1}^{r} / h$ and $\beta_{2}^{r}=2 \delta_{2}^{r} / h$. The integral terms in (47) are evaluated using the LGL quadrature according to

$$
\mathbf{R}_{i, j}^{k, r}= \begin{cases}\mathbf{B}_{j, r}^{k, r} F_{i, j} & \text { if } q_{1}^{r}<k<q_{2}^{r}, \\ \frac{2-\beta_{1}^{r}}{2} \sum_{l=1}^{n+1} \mathbf{B}_{l, b}^{k, r} L_{l, j}^{b} F_{i, l}^{b} & \text { if } k=q_{1}^{r} \neq q_{2}^{r}, \\ \frac{\beta_{2}^{r}}{2} \sum_{l=1}^{n+1} \mathbf{B}_{l, c}^{k, r} L_{l, j}^{c} F_{i, l}^{c} & \text { if } k=q_{2}^{r} \neq q_{1}^{r}, \\ \frac{\beta_{2}^{r}-\beta_{1}^{r}}{2} \sum_{l=1}^{n+1} \mathbf{B}_{l, d}^{k, r} L_{l, j}^{d} F_{i, l}^{d} & \text { if } k=q_{1}^{r}=q_{2}^{r}, \\ \mathbf{0} & \text { otherwise, }\end{cases}
$$

where

$$
\mathbf{B}_{j, \alpha}^{k, r}=\mathbf{B}_{r}\left(\frac{h\left(\eta_{j}^{\alpha}+1\right)}{2}+(k-1) h\right), \quad L_{l, j}^{\alpha}=\phi_{j}\left(\eta_{l}^{\alpha}\right), \quad F_{i, l}^{\alpha}=\psi_{i}\left(\eta_{l}^{\alpha}\right) w_{l}
$$


and

$$
\eta_{j}^{\alpha}= \begin{cases}\zeta_{j} & \text { if } \alpha=a, \\ \frac{2-\beta_{1}^{r}}{2}\left(\zeta_{j}+1\right)-1+\beta_{1}^{r} & \text { if } \alpha=b, \\ \frac{\beta_{2}^{r}}{2}\left(\zeta_{j}+1\right)-1 & \text { if } \alpha=c, \\ \frac{\beta_{2}^{r}-\beta_{1}^{r}}{2}\left(\zeta_{j}+1\right)-1+\beta_{1}^{r} & \text { if } \alpha=d .\end{cases}
$$

As it is evident from Fig. 4, formula (45) with (49) gives fast convergence for the stability boundaries even in the presence of discontinuities in $\mathbf{B}(t)$. For example, for spindle speed range $\Omega \in[5000,25000] \mathrm{rpm}$, polynomial order $n=20$ already gives accurate results for radial immersion ratios $a_{\mathrm{e}} / D=0.05,0.1$ and 0.5 . For immersion ratios $a_{\mathrm{e}} / D=0.75$ and 1 , accurate calculations require slightly higher polynomial orders $(n=30$ and $n=25$, respectively). Note that, for $N=2$, the smaller the radial immersion, the longer the free vibration. For a fixed polynomial order $n$, the solution of the SE method is more accurate in the free vibration period than during cutting. This is due to that in the free vibration period the delayed terms disappear, thus a simple ordinary differential equation is solved. Consequently, the higher the ratio of the duration of the free vibration and cutting, the more accurate the solution. Also, note that there exists another solution for the treatment of discontinuities by locating the element boundaries at the discontinuity points of $\mathbf{B}(t)$ (see $\underline{\underline{17}}, \underline{\underline{3}}$ ). In contrast, this paper provides a generalized framework, which handles discontinuities without adjusting the number and the length of the elements.

\section{Results}

The proposed extension of the SE method is applied to single and two DoF models of milling processes taken from $\underline{\underline{10}}, \underline{\underline{5}, 6,} \underline{\underline{6}, 22}, \underline{\underline{16}}, \underline{\underline{7}}, 20$. Convergence ratio of the critical characteristic multipliers and convergence of the stability boundaries are investigated and the computational time of the stability charts are compared to those available in the literature. The Matlab code used for the calculation of stability boundaries can be downloaded from http://www.mm. bme. hu/ lehotzky/IJAMT2016.

\subsection{Single DoF model}

For the single DoF model, figures 2, 4 and 5 show stability diagrams that correspond to radial immersion ratios $a_{\mathrm{e}} / D=0.05,0.1,0.5,0.75$ and 1 . Clearly, in the case of continuous $\mathbf{B}(s)$ (such as for full-immersion milling), formulas with and without the treatment of discontinuities give the same results. The figures show that polynomial orders $n=20 \sim 30$ give accurate results for the stability boundaries on the domain $\Omega \in[5000,25000] \mathrm{rpm}$. It is also interesting to note that for lower spindle speeds higher polynomial order $(n)$ is required to achieve the same accuracy. This can be seen in Figure 5, where the stability map of full immersion downmilling is depicted for lower spindle speed ranges. It can also be inferred that longer non-zero continuous part of $\mathbf{B}(s)$ (that is longer time within the cut) requires slightly higher polynomial order $n$ for accurate results.

In order to show the efficiency of the extended SE method, the computational time of constructing stability diagrams is compared to those in the literature. For this purpose we selected cases

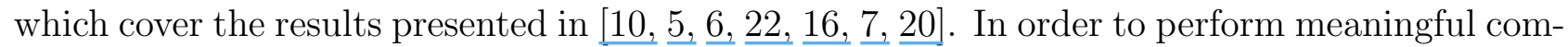
parisons, the stability diagrams were obtained using a computer with similar specifications to the computers used in the above references. Namely, a PC running Matlab 2009 with $2.1 \mathrm{GHz}$ Core 2 Duo processor and 2GB RAM memory. The consistency of the computational hardware allows performing direct comparisons between our approach and other prominent methods in 

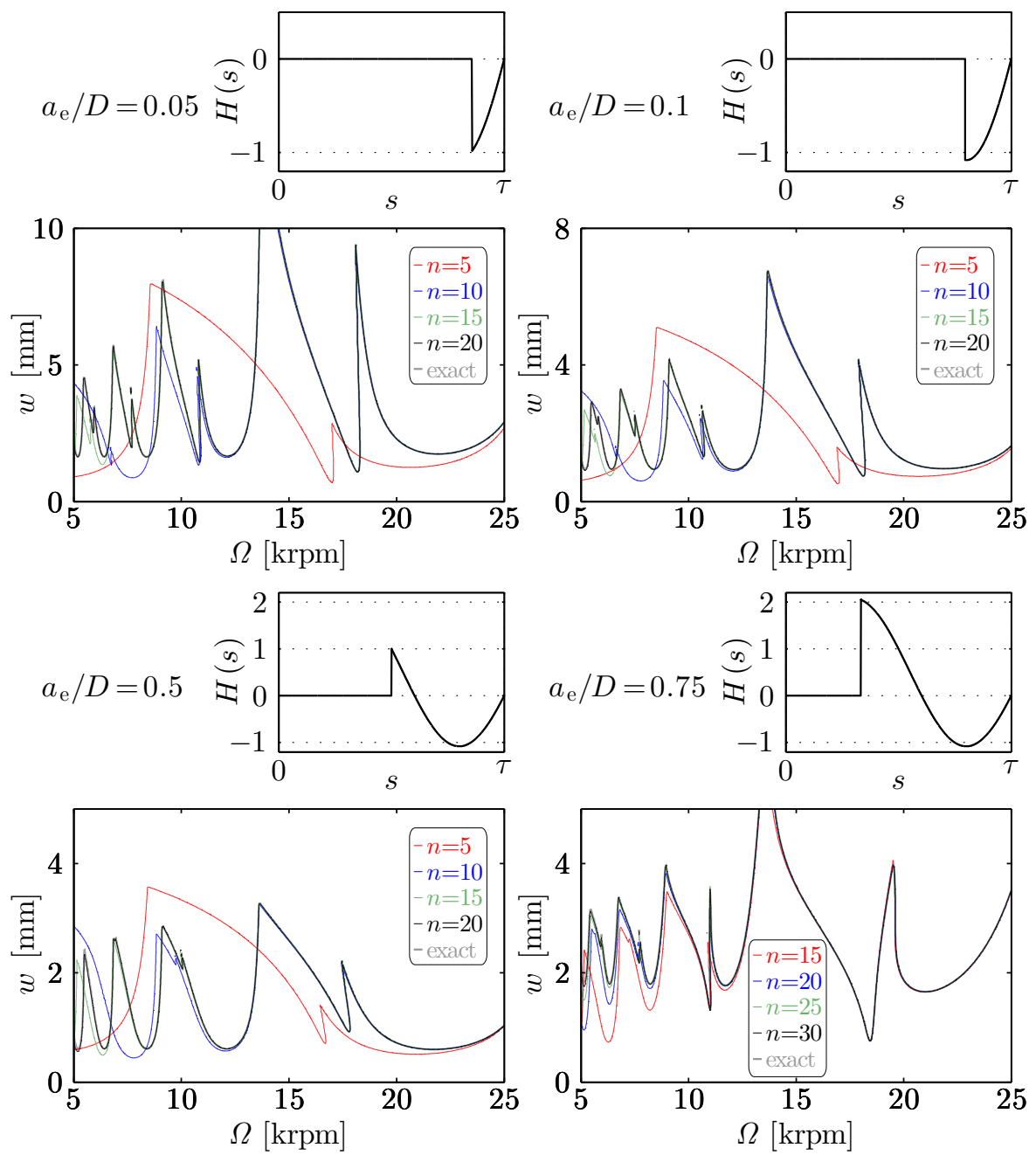

Figure 4: Convergence of stability maps for $m=1$ DoF down-milling operation with the treatment of discontinuities in $\mathbf{B}(s)$ for radial immersion ratios $a_{\mathrm{e}} / D=0.05,0.1,0.5,1$. The element number is $E=1$ and the stability maps are calculated on a $400 \times 400$ grid. Further parameters are taken from Table 1 .

the literature as shown in Table 2. Specifically, Table 2 summarizes the computational times corresponding to these comparisons. In this table, the lowest computational times are collected form 10, 5, 6, 22, 16, 7, 20 for a comparison with the SE method. In the references different spindle speed domains and immersion ratios were investigated on different grids of the parameter plane. These are all specified in Table 2 in order to facilitate precise and fair comparison. The domains for the depth of cut $w$ are selected according to the corresponding stability maps in Figures 2, 4-5 and 8. These figures were also used for the selection of polynomial order $n$ in Table 2, where polynomial orders corresponding to accurate results on the given spindle speed ranges were applied.

Table 2 shows that the SE method is the least computationally extensive approach in comparison to most of the other methods. One reason for the low computational time of the SE method is that only one matrix inversion and one matrix multiplication is needed to obtain the monodromy matrix. This contrasts the need for multiple matrix inversions which is necessary, for example, when using the semi- and full-discretization methods.

Another reason for the efficiency of the SE method is its spectral convergence rate with respect to polynomial order $n$. The spectral convergence rate is shown in Figure 6, where the absolute error of the critical characteristic multiplier with respect to a reference characteristic multiplier 

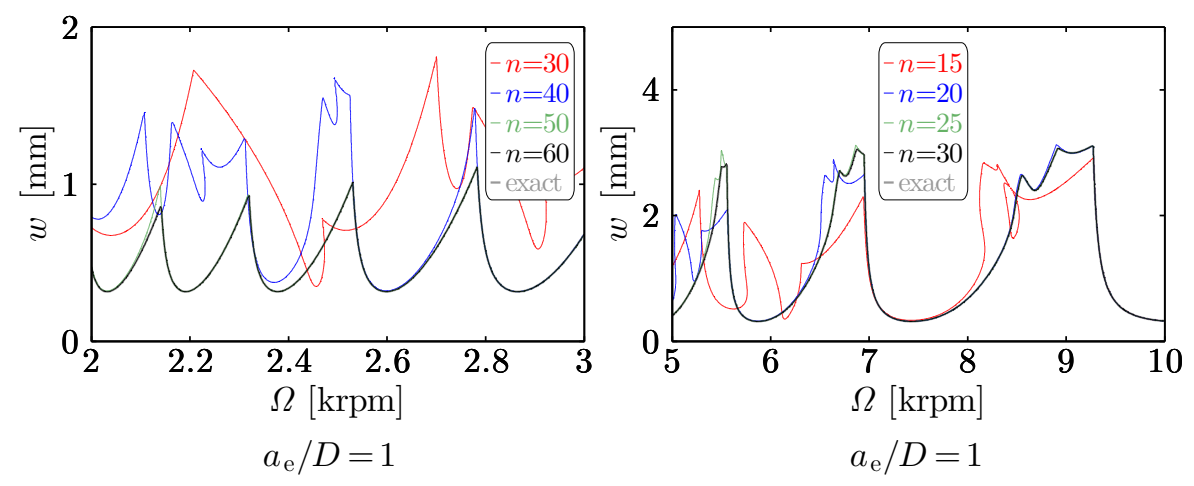

Figure 5: Convergence of stability maps for $m=1$ DoF down-milling operation with the treatment of discontinuities in $\mathbf{B}(s)$ for low spindle speed ranges. The radial immersion ratio is $a_{\mathrm{e}} / D=1$, the element number is $E=1$ and the stability maps are calculated on a $400 \times 400$ grid. Further parameters are taken from Table 1.

$\mu_{\mathrm{cr}}^{*}$ is depicted as a function of polynomial order $n$ for fixed number of elements $E$. The reference characteristic multiplier was calculated using the SE method with $E=10$ and $n=50$. It can be seen in the figure that the absolute error is progressively decreasing with the increase of polynomial order $n$ on a logarithmic scale. Comparing the convergence rates in $\underline{\underline{6}, 16,22, ~ 17,20,}$ with the corresponding rates for the SE method in Figure 6 shows that the latter converges faster with respect to $n$. The convergence rates of the SE method are similar to those in 8 . However, since the PC used for obtaining the results in 8 has better computational power, the corresponding efficiency of calculations cannot be meaningfully compared to the rest of the methods in Table 2.

The only case in Table 2 where the computational time is smaller than that of the SE method is the low immersion single degree of freedom milling with $a_{\mathrm{e}} / D=0.05$. Here, the numerical integration method 7] utilizes the closed form solution during the free oscillation. In contrast, our method does not distinguish between free oscillation and cutting, and solves the free oscillation using the SE method. Note that the separation of free vibration could also be applied to the SE method in order to decrease the computation time, however, our goal was to give a general framework rather than utilizing particular properties of the system. To elaborate, free vibration is present only for a tool with low number of teeth in case of low-radial-immersion milling. Nevertheless, the computation time for full-immersion milling (where there is no free oscillation) is less using the SE method than the numerical integration method.

Note that the SE method has two approximation parameters. One is the above discussed polynomial order $n$, the other one is the number of elements $E$. In Figure 7, the convergence rate of the absolute error of the critical characteristic multiplier is depicted on a logarithmic scale as a function of the number of elements $E$ for different polynomial orders $n$. It can be seen that the method does not have spectral convergence with respect to $E$.

\subsection{Two DoF model}

The convergence of the stability maps for the two DoF model $(m=2)$ are shown in Figure 8 for up-milling operations with different radial immersion ratios. The calculations are carried out with different polynomial orders $n$. It can be seen that polynomial order $n=20$ already provides accurate results over spindle speed domain $\Omega \in[5000,25000] \mathrm{rpm}$ for all radial immersion ratios. The computational times for different computational arrangements can be found in Table 2 ,

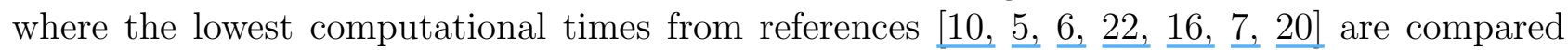
to the results of the SE method. The results show that the SE method provides the fastest 


\begin{tabular}{|c|c|c|c|c|c|c|c|c|}
\hline \multirow{3}{*}{ Grid } & \multirow{3}{*}{$\Omega \in$} & \multirow{3}{*}{$a_{\mathrm{e}} / D$} & \multicolumn{3}{|c|}{1 DoF model } & \multicolumn{3}{|c|}{2 DoF model } \\
\hline & & & \multicolumn{2}{|c|}{ SE method } & Reference & \multicolumn{2}{|c|}{ SE method } & Reference \\
\hline & & & $\mathrm{n}$ & \multicolumn{2}{|c|}{ Computational time $[\mathrm{s}]$} & $\mathrm{n}$ & \multicolumn{2}{|c|}{ Computational time $[\mathrm{s}]$} \\
\hline \multirow{4}{*}{ 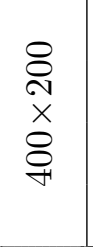 } & \multirow{4}{*}{ 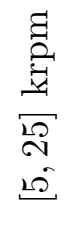 } & 0.05 & 20 & 32.35 & $13.9[7]$ & 20 & 174.30 & $960.6[5$ \\
\hline & & 0.1 & 20 & 31.96 & 293.1 [5] & 20 & 177.92 & 954.1 [5] \\
\hline & & 0.5 & 20 & 32.32 & & 20 & 136.51 & \\
\hline & & 1 & 25 & 54.49 & $129.6[7]$ & 20 & 138.49 & \\
\hline \multirow{4}{*}{$\begin{array}{l}\stackrel{8}{\odot} \\
\times \\
\stackrel{1}{8} \\
\stackrel{\odot}{\circ}\end{array}$} & \multirow{4}{*}{ 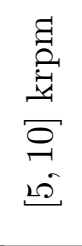 } & 0.05 & 20 & 8.78 & & 20 & 42.44 & \\
\hline & & 0.1 & 20 & 8.57 & & 20 & 41.45 & \\
\hline & & 0.5 & 20 & 8.56 & & 20 & 33.63 & \\
\hline & & 1 & 25 & 14.71 & 49 [16] & 20 & 32.76 & \\
\hline \multirow{4}{*}{$\begin{array}{l}\stackrel{10}{10} \\
\stackrel{1}{8} \\
\stackrel{8}{-}\end{array}$} & \multirow{4}{*}{ 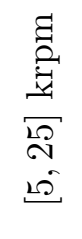 } & 0.05 & 20 & 2.41 & & 20 & 11.31 & $26.5[20]$ \\
\hline & & 0.1 & 20 & 2.44 & & 20 & 11.16 & \\
\hline & & 0.5 & 20 & 2.46 & & 20 & 8.94 & $9.3[20$ \\
\hline & & 1 & 25 & 4.04 & & 20 & 8.69 & 27.4 [20] \\
\hline
\end{tabular}

Table 2: Computational times for different milling models for different radial immersion ratios on three different grids and different spindle speed ranges, with a total number of elements $E=1$. Further parameters are taken from Table 1. For the single DoF model down-milling for the two DoF model up-milling was considered.

computational times among all of the investigated cases.

\section{Conclusions}

This paper presented an efficient application of the SE method to the stability analysis of single and two DoF models of machine tool chatter in milling operations. It was shown that discontinuities in the time-dependent parameters of the governing equations deteriorate the convergence rate of the spectral element method. In order to maintain the otherwise high convergence rate of the SE method, the method was improved for the discontinuous cases. Compact formulas were given for the construction of the monodromy matrix, which facilitates the algorithmic application of the method. It was shown through numerical examples that the extended SE method is computationally more efficient than the methods known in the machining literature.

\section{Acknowledgement}

This work was partially supported by the Hungarian National Science Foundation under grant OTKAK105433. The research leading to these results has received funding from the European Research Council under the European Unions Seventh Framework Programme (FP/2007-2013) / ERC Advanced Grant Agreement n. 340889. 


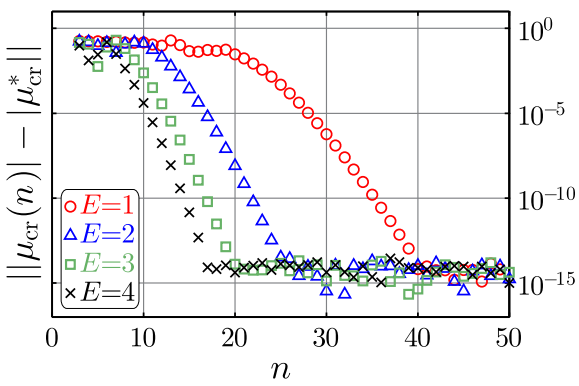

$w=0.2 \mathrm{~mm}$

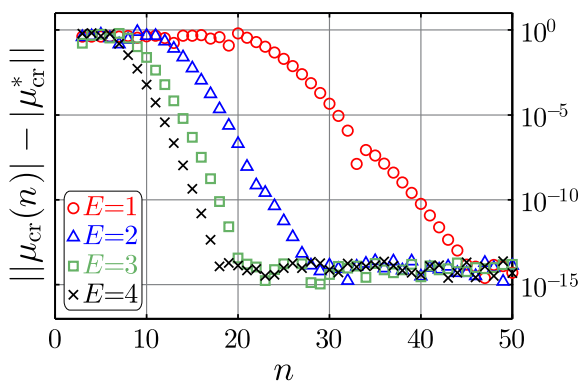

$w=1 \mathrm{~mm}$

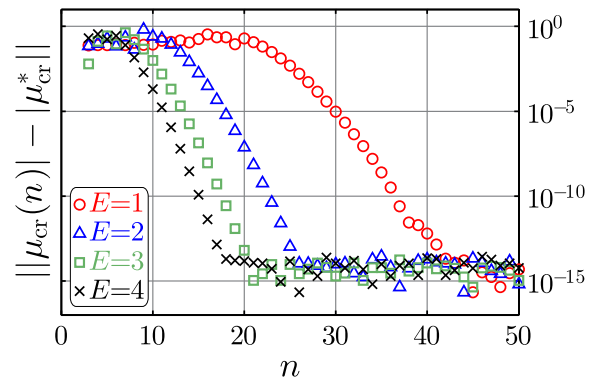

$w=0.5 \mathrm{~mm}$

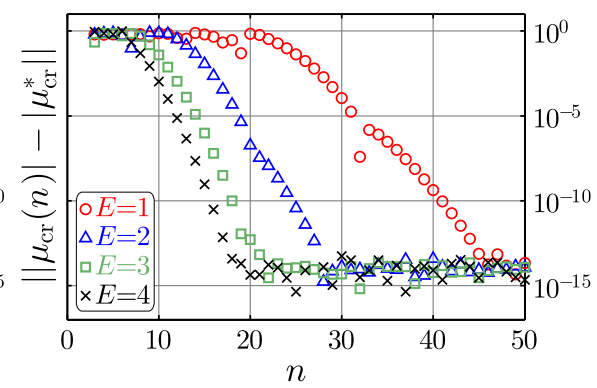

$w=1.5 \mathrm{~mm}$

Figure 6: Absolute error of the critical characteristic multiplier with respect to the reference critical multiplier $\mu_{\mathrm{cr}}^{*}$ calculated for the case $E=10$ and $n=50$. The absolute error is shown as a function of polynomial order $n$ for different element numbers $E$. Results are depicted for $m=1$ DoF down-milling operations with radial immersion ratio $a_{\mathrm{e}} / D=1$, spindle speed $\Omega=5000 \mathrm{rpm}$ and depths of cut $w=0.2, w=0.5 \mathrm{~mm}, w=1 \mathrm{~mm}$ and $w=1.5 \mathrm{~mm}$. Further parameters are taken from Table 1.

\section{Appendices}

\section{Appendix A Legendre-Gauss-Lobatto quadrature}

The Legendre-Gauss-Lobatto quadrature using $n+1$ nodes gives exact results for all polynomials with maximum order $2 n-1$. Quadrature nodes $\zeta_{j}$ are the roots of $\left(1-\zeta^{2}\right) P_{n}^{\prime}(\zeta)$, that is -1 , 1 and the roots of the first derivative of the Legendre polynomial of order $n$. The quadrature weights are given by

$$
w_{j}= \begin{cases}\frac{2}{n(n+1)} & \text { if } j=1, n+1 \\ \frac{2}{n(n+1) P_{n}^{2}\left(\zeta_{j}\right)} & \text { if } j=2,3, \ldots, n .\end{cases}
$$

\section{Appendix B Legendre polynomials}

The recursive formulas of Legendre polynomials are

$$
\begin{aligned}
& \psi_{1}(\zeta)=1 \\
& \psi_{2}(\zeta)=\zeta \\
& \psi_{j}(\zeta)=\frac{2 j-1}{j} \zeta \psi_{j-1}(\zeta)-\frac{j-1}{j} \psi_{j-2}(\zeta) \quad j=3,4, \ldots
\end{aligned}
$$

\section{References}

[1] Altintas, Y., Budak, E.: Analytical prediction of stability lobes in milling. CIRP Annals Manufacturing Technology 44, 357-362 (1995) 


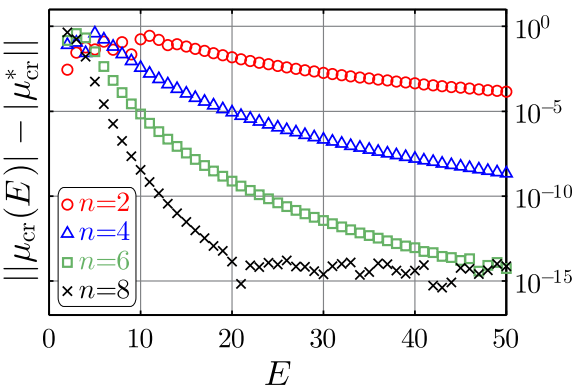

$w=0.2 \mathrm{~mm}$

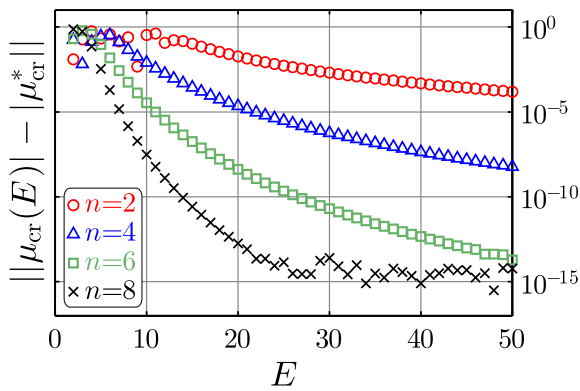

$w=1 \mathrm{~mm}$

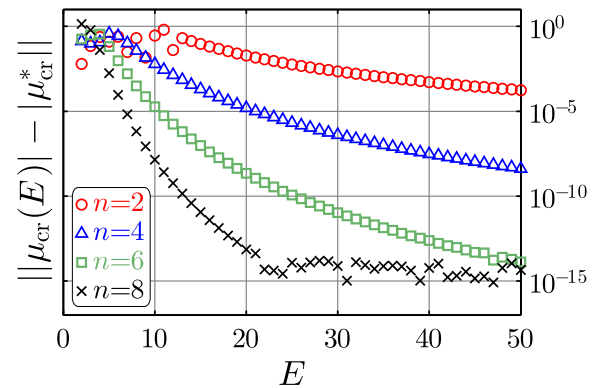

$w=0.5 \mathrm{~mm}$

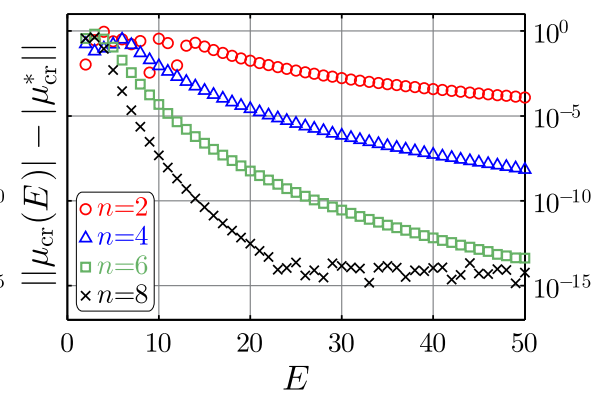

$w=1.5 \mathrm{~mm}$

Figure 7: Absolute error of the critical characteristic multiplier with respect to the reference critical multiplier $\mu_{\mathrm{cr}}^{*}$ calculated for the case $E=10$ and $n=50$. The absolute error is shown as a function of element number $E$ for different polynomial orders $n$. Results are depicted for $m=1$ DoF down-milling operation with radial immersion ratio $a_{\mathrm{e}} / D=1$, spindle speed $\Omega=5000 \mathrm{rpm}$ and depths of cut $w=0.2, w=0.5 \mathrm{~mm}, w=1 \mathrm{~mm}$ and $w=1.5 \mathrm{~mm}$. Further parameters are taken from Table 1.

[2] Bachrathy, D., Stepan, G.: Improved prediction of stability lobes with extended multi frequency solution. CIRP Annals Manufacturing Technology 62, 411-414 (2013)

[3] Bobrenkov, O.A., Khasawneh, F.A., Butcher, E.A., Mann, B.P.: Analysis of milling dynamics for simultaneously engaged cutting teeth. Journal of Sound and Vibration 329(5), $\underline{585-606(2010)}$

[4] van Dijk, N.J.M., van de Wouw, N., Doppenberg, E.J.J., Oosterling, H.A.J., Nijmeijer, H.: Robust active chatter control in the high-speed milling process. IEEE Transactions on Control Systems Technology 20(4), 901-917 (2012)

[5] Ding, Y., Zhu, L.M., Zhang, X.J., Ding, H.: A full-discretization method for prediction of milling stability. International Journal of Machine Tools and Manufacture 50, 502-509 $\underline{(2010)}$

[6] Ding, Y., Zhu, L.M., Zhang, X.J., Ding, H.: Second-order full discretization method for milling stability prediction. International Journal of Machine Tools and Manufacture 50, $\underline{926-932(2010)}$

[7] Ding, Y., Zhu, L.M., Zhang, X.J., Ding, H.: Numerical integration method for prediction of milling stability. Journal of Manufacturing Science and Engineering 133(031005), 1-9 $\underline{(2011)}$

[8] Ding, Y., Zhu, L.M., Zhang, X.J., Ding, H.: Stability analysis of milling via the differential quadrature method. Journal of Manufacturing Science and Engineering 135(044502), 1-7 $\underline{(2013)}$ 

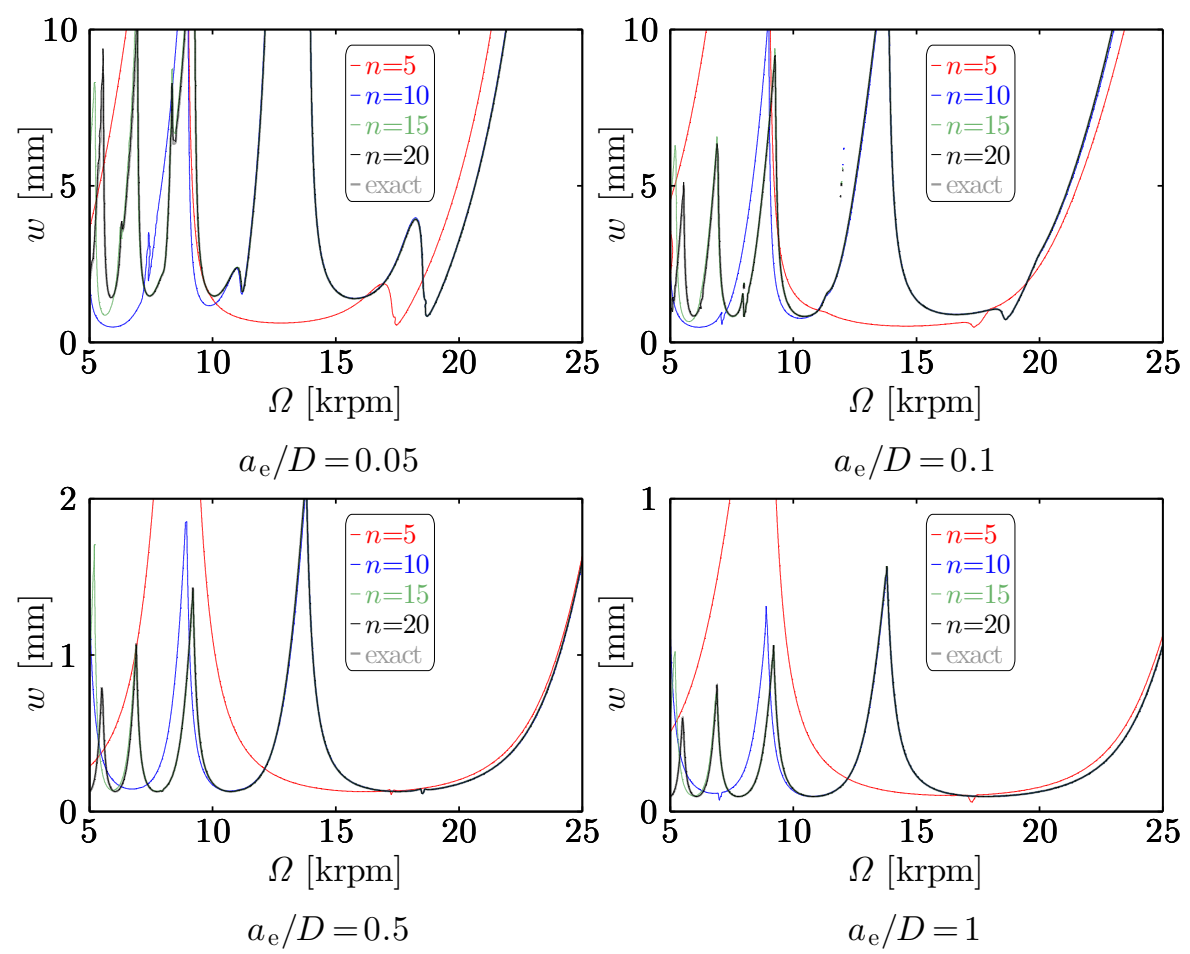

Figure 8: Convergence of stability maps for $m=2$ DoF up-milling operation with the treatment of discontinuities in $\mathbf{B}(s)$ for radial immersion ratios $a_{\mathrm{e}} / D=0.05,0.1,0.5,1$. The element number is $E=1$ and the stability maps are calculated on a $400 \times 400$ grid. Further parameters are taken from Table 1.

[9] Insperger, T., Stepan, G.: Semi-discretization method for delayed systems. International Journal for Numerical Methods in Engineering 55(5), 503-518 (2002)

[10] Insperger, T., Stepan, G.: Updated semi-discretization method for periodic delaydifferential equations with discrete delay. International Journal for Numerical Methods in Engineering 61, 117-141 (2004)

[11] Insperger, T., Stepan, G.: Semi-discretization for time-delay systems. Springer, New York (2011)

[12] Khasawneh, F.A., Mann, B.P.: A spectral element approach for the stability of delay systems. International Journal for Numerical Methods in Engineering 87, 566-592 (2011)

[13] Khasawneh, F.A., Mann, B.P.: Stability of delay integro-differential equations using a spectral element method. Mathematical and Computer Modelling 54, 2493-2503 (2011)

[14] Khasawneh, F.A., Mann, B.P.: A spectral element approach for the stability analysis of time-periodic delay equations with multiple delays. Communications in Nonlinear Science and Numerical Simulation 18, 2129-2141 (2013)

[15] Lehotzky, D., Insperger, T., Stepan, G.: Extension of the spectral element method for stability analysis of time-periodic delay-differential equations with multiple and distributed delays. Communications in Nonlinear Science and Numerical Simulation 35, 177-189 $\underline{(2016)}$

[16] Liu, Y., Zhang, D., Wu, B.: An efficient full-discretization method for prediction of milling stability. International Journal of Machine Tools and Manufacture 63, 44-48 (2012) 
[17] Mann, B.P., Young, K.A., Schmitz, T.L., Dilley, D.N.: Simultaneous stability and surface location error predictions in milling. Journal of Manufacturing Science and Engineering 127, 446-453 (2005)

[18] Mei, D., Kong, T., Shih, A.J., Chen, Z.: Magnetorheological fluid-controlled boring bar for chatter suppression. Journal of Materials Processing Technology 209, 1861-1870 (2009)

[19] Munoa, J., Mancisidor, I., Loix, N., Uriarte, L.G., Barcena, R., Zatarain, M.: Chatter suppression in ram type travelling column milling machines using a biaxial inertial actuator. CIRP Annals - Manufacturing Technology 62, 407-410 (2013)

[20] Niu, J.B., Ding, Y., Zhu, L.M., Ding, H.: Runge-kutta methods for a semi-analytical prediction of milling stability. Nonlinear Dynamics 76, 289-304 (2014)

[21] Quintana, G., Ciurana, J.: Chatter in machining processes: a review. International Journal of Machine Tools and Manufacture 51, 363-376 (2011)

[22] Quo, Q., Sun, Y., Jiang, Y.: On the accurate calculation of milling stability limits using third-order full-discretization method. International Journal of Machine Tools and Manufacture 62, 61-66 (2012)

[23] Sims, N.D.: Vibration absorbers for chatter suppression: A new analytical tuning methodology. Journal of Sound and Vibration 301, 592-607 (2007)

[24] Yang, Y., Munoa, J., Altintas, Y.: Optimization of multiple tuned mass dampers to suppress machine tool chatter. International Journal of Machine Tools and Manufacture $\underline{\mathbf{5 0}, 834-842(2010)}$

[25] Zhao, M.X., Balachandran, B.: Dynamics and stability of milling process. International Journal of Solids and Structures 38, 2233-2248 (2001) 